\title{
Optimized Sample Centering for Best Your Experimental Results
}

\author{
Martin Adam $^{1}$, Michael Ruf ${ }^{2}$ and Jaap Toorn ${ }^{1}$ \\ ${ }^{1}$ Bruker AXS GmbH, Oestliche Rheinbrueckenstrasse 49, 76187 Karlsruhe, GERMANY \\ ${ }^{2}$ Bruker AXS Inc., 5465 E. Cheryl Parkway, Madison, WI 53711, USA \\ E-mail: Martin.Adam@Bruker.com
}

Recent improvements in X-ray instrumentation allow the investigation of ever smaller crystals. Today's samples are typically in a range of less than $100 \mu \mathrm{m}$ in size, sometimes even less than $10 \mu \mathrm{m}$. These small crystals require mechanically stable goniometers with the best possible sphere of confusion. All our goniometers possess a sphere of confusion smaller than $7 \mu \mathrm{m}$. However, special attention must be paid on a proper alignment of the sample since a poorly aligned sample leads to not optimal data sets.

To accomplish the optical alignment video microscopes typically equipped with electronic crosshairs became the standard on modern single crystal diffraction systems. The center of the crosshairs needs perfectly to match with the center of the goniometer and the crystal needs to be perfectly centered in the crosshairs. The latter is often prevented by samples providing little optical contrast, resulting in a significant error. As a consequence the crystals often oscillates in the beam or-in the worst casemoves completely out of the beam during the data collection. While scaling programs, such as SADABS, can correct for an oscillation of the sample, there is no way to compensate for information lost when the crystal is out of the beam as there is simply no diffraction signal recorded. An there is a second problem, even with the best aligned instrument and the best optically aligned sample there is no way to identify the best diffracting area within a sample or the least pathologic area within a pathologically twinned or disordered sample.

Today, we present a new package AGH II designed for the best possible alignment of the a given sample. The system consists of soft- and hardware to eliminate the shortcoming describes above.

- The AGH software takes advantage of a newly developed Automated Goniometer Head (AGH), which allows a precise scans of the sample in $\mathrm{X}, \mathrm{Y}$ and $\mathrm{Z}$ direction. The moving the sample is exposed to X-rays while collecting up to 70 images per second with the PHOTON II detector.

- The images taken in each direction are automatically analyzed for diffraction quality. From the best quality images the position of the AGH during the scan is identified and feed back to the AGH for the usage in the diffraction experiment.

- For samples decomposing in the beam the setup allows the regular usage of "fresh" segment of the crystal. Particularly protein diffraction experiments benefit from this approach. The new software engine if fully incorporated into the PROTEUM3 and APEX3 software suites.

The new AGH can be mounted on our KAPPA or the FIXED-CHI goniometer. However-in contrast to other automated goniometer heads in the market or in use at synchrotrons - the AGH complies with the IUCR requirements for goniometer heads and therefore, it can be mounted on many others goniometers. The magnet base of the AGH is ideal for the use with sample pins (typical height $22 \mathrm{~mm}$ ). Highly reliable, encoder controlled Piezo motors not only ensure high mechanical stability but also guarantee a long lifetime of the new tools. 